\section{TO THE EDITOR}

\section{Role of Midazolam in Parkinsonian Tremors: To Use or Not to Use}

Resting tremors are one of the extremely disabling cardinal symptoms of Parkinson's disease (PD). The intraoperative precipitation of such a symptom during deep brain stimulation can impose many challenges for anesthesiologist as well as for the surgeon. Here we have highlighted the effect of midazolam during such an event.

Case 1- A 82-year-old patient with PD was posted for the battery replacement under monitored anesthesia care with remifentanil (0.05 mcg / $\mathrm{kg} / \mathrm{min})$ and propofol $(25 \mathrm{mcg} / \mathrm{kg} /$ min) infusion. He had previously undergone deep brain stimulation (DBS) with bilateral sub thalamic nuclei (STN) electrode insertion and battery placement. During the skin closure, patient started tremors on left hand (same side of surgery). Though the patient was completely sedated at the time, $0.5 \mathrm{mg}$ of intravenous midazolam was given and surprisingly tremors were abolished within a few minutes.

Case 2- A 43-year-old otherwise healthy PD patient was scheduled for DBS with bilateral STN electrode insertion and battery placement. Initial part of procedure was done in remifentanil (0.05 $\mathrm{mcg} / \mathrm{kg} / \mathrm{min})$ and propofol $(25 \mathrm{mcg} / \mathrm{kg} /$ min) infusion. She suddenly started tremors on left hand during left side electrode insertion. In this event too, patient was calm and mildly sedated. As these movements were distracting the surgical field, $0.5 \mathrm{mg}$ of intravenous midazolam was given. Interestingly, hand tremors aborted within a few minutes.

The resting tremors of PD may precipitate during surgical stress or anxiety. ${ }^{1}$ Though both patients seemed to be calm and mildly sedated, subtle surgical stress and anxiety coupled with off medications for prolonged time, might precipitate this distressing symptom. Occurrence of intraoperative tremors can interfere with patient's monitoring (electrocardiography, pulse oximeter and blood pressure measurement), chances of decannulation of intravenous or arterial lines and importantly slight movement at surgical field can distract the electrode insertion and produce catastrophic complications. The pathogenesis of PD tremors is a complex mechanism and still not fully understood.
The proposed mechanisms include the dopamine - nor adrenaline interactions at substantia nigra, cerebello-thalamic circuit activation and role of locus coeruleus. ${ }^{2,3}$ In animal experiments, midazolam has shown to produce its effect on many of the above mentioned sites or pathways thus likely to interfere with tremor genesis and treatment. ${ }^{4}$ On the other hand, some reports suggested that midazolam used for sedation may augment or even precipitate extra pyramidal symptoms. ${ }^{5}$ However, these cases were found in normal patients without PD. Thus it is likely that midazolam may produce different forms of interactions at different structures of brain of patients with or without PD. This hypothesis needs further research.

In conclusion, intraoperative tremors can be precipitated even in calm or sedated patients with PD. Low dose midazolam is effective to abort such an episode and hence may be used as first alternative.

Tumul Chowdhury, Ronald B. Cappellani, Stephen E. Kowalski Department of Anesthesiology and Perioperative Medicine University of Manitoba, Winnipeg, Manitoba, Canada Email:tumulthunder@gmail.com

\section{REFERENCES}

1. Schlesinger I, Benyakov O, Erikh I, et al. Parkinson's disease tremor is diminished with relaxation guided imagery. Mov Disord. 2009;24:2059-62.

2. Isaias IU, Marzegan A, Pezzoli G, et al. A role for locus coeruleus in Parkinson tremor. Front Hum Neurosci. 2011;5:179.

3. Helmich RC, Hallett M, Deuschl G, et al. Cerebral causes and consequences of parkinsonian resting tremor: a tale of two circuits? Brain. 2012;135:3206-26.

4. Laurent JP, Mangold M, Humbel U, et al. Reduction by two benzodiazepines and pentobarbitone of the multiunit activity in substantia nigra, hippocampus, nucleus locus coeruleus and nucleus raphé dorsalis of encéphale isolé rats. Neuropharmacology. 1983;22:501-11.

5. Vorsanger GJ, Roberts JT. Midazolam-induced athetoid movements of the lower extremities during epidural anesthesia reversed by physostigmine. J Clin Anesth. 1993;5:494-6.

\section{TO THE EDITOR}

\section{CT Perfusion in the Management of Acute Stroke}

The phrase "time equals brain" is a well established mantra in stroke medicine. The quest to prolong the therapeutic window is just as crucial as identifying the appropriate patient population in which to begin the definitive therapy that can be fraught with hazards. It is well established that thrombolytic therapy can significantly reduce morbidity and mortality within a 4.5 hour window for some stroke patients ${ }^{1}$. The determination of this patient group is not as clear cut. In addition to evaluating the patient clinically, the choice of diagnostic imaging can be confusing to practitioners as the technology continues to evolve at a pace faster than our understanding of the disease. The most up to date guidelines by the American Stroke Association ${ }^{2}$ and the Canadian Stroke Strategy Best Practices and Standards Writing Group ${ }^{3}$ both agree that obtaining a non-enhanced head $\mathrm{CT}$ and when available and indicated, MRI diffusion weighted and fluid attenuated inversion recovery sequences. Until this writing, CT Perfusion (CTP) investigations are absent from the Canadian recommendations and the Americans only recommend CTP for cases presenting more than three hours after onset.

In a retrospective institutional survey ${ }^{4}$, we reviewed the clinical management decisions with one vascular neurologist and one general neurologist (who covers the stroke service on call) for 15 patients who presented with acute stroke and had CT 